\title{
EXPERIENCIA DE EVALUACIÓN FORMATIVA EN UNA UNIDAD DIDÁCTICA DE BODY-COMBAT
}

Experience of formative assessment in a Body-combat lesson plan

Experiência de avaliação formativa em um plano de aula Body-combat

\section{Mario Sobejano Carrocera}

Instituto de Enseñanza Secundaria 9 Valles, Cantabria, España. Teléfono:

+34620920886. Correo electrónico: mario.sobejano@gmail.com

\section{Resumen}

El fin último de la materia de Educación física en la ESO y el Bachillerato es generar en el alumnado hábitos de práctica de actividad física saludable en su tiempo de ocio. La unidad didáctica de body-combat busca presentar a los alumnos una alternativa de actividad física ofertada en muchos centros de fitness en la actualidad. De igual manera se pretende desarrollar unos contenidos vinculados a la expresión corporal, una de las manifestaciones motrices menos practicadas por el alumnado en su tiempo libre y de ocio. En todo el proceso de enseñanza aprendizaje de esta unidad los instrumentos de evaluación formativa proporcionados constituyen una herramienta fundamental para guiar al alumnado en el diseño de una coreografía grupal de manera autónoma. Los buenos resultados finales del grupo-clase en la unidad planteada demuestran la utilidad de las estrategias evaluativas desde el punto de vista formativo. No obstante no se ha constatado si los alumnos han adoptado este tipo de prácticas en su tiempo libre y de ocio. Este aspecto podría ser contemplado en futuras investigaciones.

Palabras clave: Evaluación formativa; actividad física; expresión corporal; bodycombat

\begin{abstract}
The ultimate goal of Physical Education in high school is to generate habits of healthy physical activity in students during their leisure time. The body-combat lesson plan seeks to present students with an alternative of physical activity offered in many fitness centers today. In the same way it is intended to develop content linked to the corporal expression, one of the motor manifestations less practiced by the students in their free time and leisure. Throughout the teaching-learning process of this unit the provided Experiencia de Evaluación Formativa en una Unidad didáctica de Body-Combat
\end{abstract}


formative evaluation instruments constitute a fundamental tool to guide the students in the design of a group choreography in an autonomous way. The good final results of the group-class in the unit raised demonstrate the usefulness of formative evaluation strategies. However, it has not been established whether the students have adopted this type of practice in their leisure time. This aspect could be the goal in future investigations.

Keywords: Formative assessment; physical activity; body language; body-combat

\section{Resumo}

O objetivo final da educação física no ESO e bacharelado é gerar nos alunos que praticam hábitos saudáveis de atividade física no lazer. Didática unidade body-combat visa apresentar aos alunos uma atividade física alternativa oferecida em muitos centros de fitness hoje. Da mesma forma, pretende desenvolver um conteúdo relacionado à linguagem corporal, uma das demonstrações de condução menos praticados pelos alunos em seu tempo livre e lazer. Durante todo o processo de aprendizagem desta unidade de ferramentas de avaliação formativa fornecidas são uma ferramenta essencial para orientar os alunos na elaboração de uma coreografia grupo independente. Bons resultados finais do grupo classe na unidade levantada demonstrar a utilidade das estratégias de avaliação do ponto de vista da formação. No entanto, não foi apurado se os alunos adotaram tais práticas em seu lazer e entretenimento. Este aspecto pode ser referido em pesquisas futuras.

Palavras-chave: Avaliação formativo; atividade física; expressão corporal; corpo combate

\section{Introducción}

El Real Decreto 1105/2014, por el que se establece el currículo básico de la Educación Secundaria Obligatoria y del Bachillerato, identifica las situaciones de índole artística o de expresión como uno de los cinco tipos de manifestaciones motrices en los que se debe estructurar la materia de Educación Física. Actualmente, aún sigue habiendo profesorado que reconoce no impartir contenidos relacionados con la expresión corporal con su alumnado motivado por la escasa formación recibida al respecto y la ambigüedad de su evaluación (Montálvez, 2012). Por otra parte, Reverter y Barbany (2007) analizan 
la gran evolución que han sufrido los centros dedicados a la práctica de actividad física y salud en los que se ofertan multitud de actividades y productos adaptados a las necesidades de los usuarios (crossfit, body-pump, spinning, body-combat...etc).

En este sentido, el mencionado Real Decreto 1105/2014 refleja en sus anexos como en el informe Eurydice de la Comisión Europea de 2013 se constata que el 80\% de los niños y niñas en edad escolar solo practican actividad física en la escuela. Y en relación a ello, el porcentaje de chicos y chicas que practican actividad física extraescolar relacionada con la expresión corporal es menor al 20\% (Uxía, 2006).

Esta experiencia pretende aproximar al alumnado una actividad ofertada en los centros de fitness en la actualidad, con el objetivo de que conozcan una posibilidad más de utilización saludable de su tiempo libre y de ocio. Por otra parte, el uso de los instrumentos de evaluación formativa se convierte en una herramienta facilitadora de todo el proceso de enseñanza-aprendizaje tanto para el alumno como para el docente al identificar los aspectos esenciales de las producciones de los estudiantes evitando la ambigüedad en su tratamiento.

\section{Contextualización}

La propuesta se ha llevado a cabo en el único curso de primero de Bachillerato al que el docente impartía clase de Educación Física, formado por 19 alumnos. La elección de este curso para la experiencia radicaba en que tras una encuesta inicial, ninguno de los alumnos tenía experiencia previa en los contenidos que se pretendía desarrollar en la unidad.

\section{Diseño y desarrollo}

La unidad didáctica de Body-combat se desarrolla en un total de 8 sesiones (tabla 1). En las dos primeras sesiones de la unidad se lleva a cabo una explicación práctica de las principales técnicas del body-combat (jab, cross, hook, upper, front kick...etc.). Estas técnicas tendrán que ser aplicadas en el diseño de una coreografía grupal para la que se destinarán las cuatro sesiones siguientes (sesiones $3^{\mathrm{a}}$ a $6^{\mathrm{a}}$ ). En la primera de esas sesiones de trabajo grupal autónomo (sesión $3^{\mathrm{a}}$ ) se entregan a los alumnos dos instrumentos de evaluación formativa. El primero de ellos es una Escala de valoración diferenciada para una coreografía grupal (figura 1). 
Tabla 1.

Contenido de las sesiones

\begin{tabular}{ll}
\hline & \multicolumn{1}{c}{ Columna 1 } \\
\hline Sesión $1^{\mathrm{a}}$ y $2^{\mathrm{a}}$ & Presentación y aprendizaje de las principales técnicas del body-combat \\
Sesión $3^{\mathrm{a}}$ a $6^{\mathrm{a}}$ & Preparación del trabajo grupal (coreografía) \\
Sesión 7 & Grabación de autoevaluación \\
Sesión $8^{\mathrm{a}}$ & Calificación final \\
\hline
\end{tabular}

ESCALA DE VALORACIÓN DE UNA COREOGRAFIA DE BODY-COMBAT

\begin{tabular}{|c|c|}
\hline GRUPO AL QUE SE LE EVALUA & \\
\hline \multicolumn{2}{|l|}{ CRITERIO A VALORAR } \\
\hline \multicolumn{2}{|l|}{ PASOS DE LA COREOGRAFIA } \\
\hline La coreografia incluye más de 5 pasos vistos en clase (jab, cross, descendent elbow, front kick, jumping jack... etc) & 30 \\
\hline La coreografia incluye entre 4 y 5 pasos de los vistos en clase & 20 \\
\hline La coreografia incluye 3 pasos de los vistos en clase. & 10 \\
\hline La coreografia incluye menos de 3 pasos vistos en clase & 5 \\
\hline \multicolumn{2}{|l|}{ ESTRUCTURA DE LA COREOGRAFIA } \\
\hline La coreografia tiene fases diferenciadas en las que se utilizan ambas guardias y presenta distintas intensidades & 30 \\
\hline La coreografia tiene fases diferenciadas en las que se utilizan ambas guardias pero no se marcan distintas intensidades & 20 \\
\hline La coreografia no presenta fases diferenciadas ni los ejercicios se ejecutan con distintos nivel de intensidad & 10 \\
\hline Apenas se observa estructura y simplemente son pasos que se ejecutan de manera continuada sin ninguna relación entre ellos (repetición) & 5 \\
\hline \multicolumn{2}{|l|}{ COORDINACIÓN DE LA COREOGRAFIA CON LA MÚSICA } \\
\hline La coreografía se adapta perfectamente al ritmo de la música en su totalidad cambiando su velocidad cuando la música to hace. & 30 \\
\hline La coreografia solamente se adapta en algún momento de ta música. & 15 \\
\hline La coreografia no guarda casi ninguna relación con ta música elegida y apenas se adaptan al ritmo de la misma. & 5 \\
\hline \multicolumn{2}{|l|}{ COORDINACIÓN DEL GRUPO } \\
\hline Casi todo el grupo está coordinado durante la mayor parte de la coreografia. & 10 \\
\hline Hay descoordinación del grupo pero solamente en algunos momentos muy puntuales. & 5 \\
\hline Hay una gran descoordinación del grupo durante más de la mitad de la coreografia. & 0 \\
\hline
\end{tabular}

Figura 1.

Escala de Valoración de una Coreografía Grupal de Body-Combat (elaboración propia).

El segundo consiste en una escala de autoevaluación individual para la misma coreografía (figura 2). En ambos instrumentos se recogen los principales aspectos que determinarán la calificación final del producto, es decir de la coreografía diseñada. En la sesión $7^{\mathrm{a}}$ se graban con una tablet las coreografías de los distintos grupos con el fin de realizar una evaluación previa que permita revisar los aspectos de última hora, dejando la última de las clases (sesión 8ª) para la calificación final.

\section{Evaluación}

La evaluación se lleva a cabo a partir de la reproducción de los vídeos grabados de las distintas coreografías. En esta dinámica, cada uno de los miembros del grupo evaluado, realiza una auto-calificación individual utilizando el instrumento compartido al inicio de 
la unidad. El resto de grupos realizan co-calificaciones intergrupales. La calificación final de la unidad viene determinada por tres instrumentos fundamentalmente: la escala de valoración para la coreografía grupal, para la que se hace la media de las cocalificaciones de los grupos, conjuntamente con las referencias del profesor (heterocalificación), siempre que no difieran en más de un 20\%, (se consideró de manera consensuada con el alumnado que un porcentaje superior podría deberse a motivos “menos objetivos" como falta de atención, diferencias personales, etc.) ; la escala de autoevaluación individual, para que la que se respeta, de igual manera, las autocalificaciones realizadas por cada uno de los alumnos junto con las del docente y finalmente el trabajo diario a partir de las anotaciones del profesor en un registro anecdótico.

\begin{tabular}{|c|c|}
\hline NOMBRE ALUMNO/A: & \\
\hline \multicolumn{2}{|l|}{ CRITERIO A VALORAR } \\
\hline \multicolumn{2}{|l|}{ EJECUCION DE LOS PASOS DE LA COREOGRAFIA } \\
\hline He ejecutado perfectamente todos los movimientos de la coreografía (posición del cuerpo, codos pegados, guardia alta, cuerpo agrupado...) & 5 \\
\hline La ejecución de los pasos la he hecho bien en general, pero en algunos movimientos, no he sido capaz de realizarlos correctamente & 3 \\
\hline La ejecución de mis movimientos no ha sido la más adecuada, puesto que me ha costado reproducir los golpes correctamente & 2 \\
\hline No en sido capaz de ejecutar ningún movimiento con cierta soltura (golpes de brazos, patadas, transiciones...etc) & 1 \\
\hline \multicolumn{2}{|l|}{ INTENSIDAD DE EJECUCIÓN EN RELACIÓN A LA MÚSICA } \\
\hline La intensidad de mis golpes ha sido perfecta diferenciando fases más relajadas con movimientos mucho más agresivos e intensos & 5 \\
\hline La intensidad de mis golpes ha sido bastante alta en general, pero no se ha combinado con otros ritmos en función de la fase de la canción & 3 \\
\hline La intensidad de mis golpes ha sido casi siempre igual y bastante relajada haciendo que los golpes no fueran muy realistas & 1 \\
\hline \multicolumn{2}{|l|}{ COORDINACIÓN DE MIS PASOS CON LA MÜSICA } \\
\hline Durante toda la coreografia he sido capaz de seguir perfectamente el ritmo de la música coordinándolo con mis movimientos. & 4 \\
\hline En ocasiones me he perdido y mis movimientos no coincidian con el ritmo que marca la canción. & 2 \\
\hline He tenido serias dificultades para seguir el ritmo de la canción y mis movimientos no coincidieron ni con mis compañeros ni con la música. & 1 \\
\hline \multicolumn{2}{|l|}{ CONOCIMIENTO DE LA COREOGRAFIA } \\
\hline Me conocía perfectamente los pasos de la coreografia y en níngún momento de la canción he dudado y/o me he perdido. & 6 \\
\hline A pesar de saberme bien los pasos de la coreografia, en alguna ocasión (1-2) me he perdido y he tenido que mirar a mis compañeros. & 4 \\
\hline He tenido que recurrir en varias ocasiones a un compañero para acordarme de los pasos, puesto que se me olvidaban. & 2 \\
\hline
\end{tabular}

Figura 2.

Escala de autoevaluación de una coreografía de Body-Combat (elaboración propia).

En cuanto a los resultados, se puede comprobar (tabla 2) que en la totalidad del alumnado fueron muy positivos siendo la calificación final mayoritaria de notable. En los intercambios orales realizados al final de la séptima sesión, el alumnado manifestó que la unidad había sido mejor de lo esperado, hecho especialmente relevante en los 
chicos, por ser un colectivo en el que los contenidos de expresión corporal suelen ser peor percibidos.

Tabla 2.

Calificaciones finales U.D Body Combat

\begin{tabular}{cccccc}
\hline & $\begin{array}{c}\text { Suspensos } \\
(\mathbf{0 - 4 , 9 )}\end{array}$ & $\begin{array}{c}\text { Aprobados } \\
(\mathbf{5 - 6 , 9 )}\end{array}$ & $\begin{array}{c}\text { Notables } \\
\mathbf{( 7 - 8 , 9 )}\end{array}$ & $\begin{array}{c}\text { Sobresalientes } \\
(\mathbf{9 - 1 0 )}\end{array}$ & Total \\
\hline Alumnos & 0 & 3 & 15 & 1 & 19 \\
$\%$ & 0 & 15.8 & 79.0 & 5.2 & 100 \\
\hline
\end{tabular}

\section{Conclusiones}

Los resultados obtenidos en la calificación final de la unidad se pueden categorizar de muy buenos, siendo la nota media del grupo-clase un 8. Los instrumentos de evaluación utilizados han permitido que el alumnado orientara sus esfuerzos en los aspectos más importantes y tomara parte activa en la calificación de sus producciones, aspecto este último, muy valorado por ellos. La unidad didáctica fue valorada de manera muy positiva incluso por los chicos durante las asambleas finales, si bien no se ha podido constatar el grado de continuidad que ha podido tener en su tiempo libre y de ocio. Este último aspecto puede ser objeto de análisis para futuras ocasiones a partir del Diario de Evidencias de Actividad Física, Sobejano, Pérez Pueyo y Hortigüela (2016).

\section{Referencias}

Montávez, M. (2012). La expresión corporal en la realidad educativa. Descripción y análisis de su enseñanza como punto de referencia para la mejora de la calidad docente en los centros públicos de educación primaria de la ciudad de Córdoba (Tesis Doctoral). Universidad de Córdoba, España. Recuperado de http:/helvia.uco.es/xmlui/bitstream/handle/10396/6310/9788469512753.pdf?sequ $\underline{\text { en }}$

Real Decreto 1105/2014, de 26 de diciembre, por el que se establece el currículo básico de la Educación Secundaria Obligatoria y del Bachillerato. (BOE núm. 3, 3 de enero de 2015).

Reverter, J., \& Barbany, J.R (2007). Del gimnasio al ocio-salud. Apunts. Educación Física y Deportes 90, 59-68. 
Sobejano, M, Pérez Pueyo, Á., \& Hortigüela, D. (2016). En contexto: Diario de evidencias de actividad física (DEAF): un nuevo instrumento de evaluación. Tandem. Didáctica de la Educación Física 52.

Uxía, I. (2006). La expresión corporal en educación física: realidad y pensamiento de profesores y alumnos. Recuperado de http://altorendimiento.com/la-expresioncorporal-en-educacion-fisica-realidad-y-pensamiento-de-profesores-y-alumnos. 